\title{
Diffraction efficiency of polarization holographic gratings recorded in azopolymer thin films coated using different solvents
}

Blaga Blagoeva, Lian Nedelchev, Georgi Mateev, Elena Stoykova, Dimana Nazarova

Blaga Blagoeva, Lian Nedelchev, Georgi Mateev, Elena Stoykova, Dimana Nazarova, "Diffraction efficiency of polarization holographic gratings recorded in azopolymer thin films coated using different solvents," Proc. SPIE 11367, Photosensitive Materials and their Applications, 113671G (1 April 2020); doi: $10.1117 / 12.2555756$ 


\title{
Diffraction efficiency of polarization holographic gratings recorded in azopolymer thin films coated using different solvents
}

\author{
Blaga Blagoeva ${ }^{1}$, Lian Nedelchev*1,2, Georgi Mateev ${ }^{1}$, Elena Stoykova ${ }^{1}$, Dimana Nazarova ${ }^{1}$ \\ ${ }^{1}$ Institute of Optical Materials and Technologies, Bulgarian Academy of Sciences, Sofia, Bulgaria \\ ${ }^{2}$ University of Telecommunications and Post, Sofia, Bulgaria \\ *lian@iomt.bas.bg
}

\begin{abstract}
We present a study of the diffraction efficiencies of polarization holographic gratings recorded in thin films of the azopolymer PAZO (poly[1-[4-(3-carboxy-4-hydroxyphenylazo) benzenesulfonamido]-1,2-ethanediyl, sodium salt]). Two series of layers have been prepared using two different solvents - distilled water and methanol. The gratings are inscribed by two plain waves with orthogonal circular polarizations (left and right circular) from a He-Cd gas laser $(442 \mathrm{~nm})$ at recording angle $20^{\circ}$, corresponding to grating period $1.3 \mu \mathrm{m}$. Higher diffraction efficiency is obtained for the thin film samples spin-coated from the methanol solution for thicknesses below $600 \mathrm{~nm}$. Diffraction efficiency higher than $27 \%$ was achieved, as well as surface relief height more than $500 \mathrm{~nm}$.
\end{abstract}

Keywords: Polarization holographic grating, Diffraction efficiency, Azopolymers, Thin films, Photoinduced birefringence

\section{INTRODUCTION}

Azopolymers are one of the most efficient materials able to register the polarization state of light. This is achieved through a series of trans-cis-trans isomerizations of the azobenzene moieties, leading to their reorientation perpendicularly to the direction of linearly polarized light ${ }^{1}$. This effect, known also as Angular Hole Burning (AHB) results on a macroscopic level formation of significant photoinduced anisotropy - birefringence and dichroism. Based on this unique property of the azobenzene-containing materials, they have been used for numerous applications - most often for reversible optical data storage $e^{2-4}$, tunable surface relief gratings ${ }^{5-7}$ or diffractive optical elements with unique polarization properties $^{8-10}$. The research on azopolymers during the last decades is also described in some milestone reviews ${ }^{11-13}$.

Most of the above-mentioned applications are related with recoding of polarization holographic grating (PHG) in an azopolymer thin film. In some cases beams with orthogonal linear polarizations are used ${ }^{14,15}$, but more often beams are set to orthogonal circular polarizations (left and right), as this leads to formation of gratings with higher diffraction efficiency ${ }^{16}$. As known, together with the polarization grating in the volume of the material, topographic grating is formed on the surface of the azopolymer film, usually denoted as surface relief grating (SRG) ${ }^{17,18}$. The SRG has the same period as the volume grating and contributes to the increase of the diffraction efficiency.

During the last decades, an azopolymer commonly used for optical applications is the commercially available poly[1-[4(3-carboxy-4-hydroxyphenylazo)benzenesulfonamido]-1,2-ethanediyl, sodium salt], often abbreviated as PAZO. An important advantage of this polymer is that it is readily soluble in water, as well as in methanol. Thus, usage of toxic organic solvents can be avoided. In some studies pure water is preferred as solvent ${ }^{19,20}$, as in other PAZO is dissolved in methanol $^{21,22}$. In our earlier studies ${ }^{23,24}$, we have compared thin films of PAZO coated from water and from methanol solution. Higher surface smoothness was reported for the PAZO/methanol layers, as well as better optical quality, even when drying occurs at increased temperature ${ }^{24}$.

In this study, we present an investigation of the properties of polarization holographic gratings and the concurrently formed surface relief gratings in series of azopolymer thin films with different thickness. One of the series is deposited from solutions of PAZO in methanol, while the other is coated from water solutions of PAZO. Kinetics of the diffraction efficiency as well as height of the surface relief topography are presented and compared.

Photosensitive Materials and their Applications, edited by Robert R. McLeod, Inmaculada Pascual Villalobos,

Yasuo Tomita, John T. Sheridan, Proc. of SPIE Vol. 11367, 113671G · (c) 2020 SPIE

CCC code: $0277-786 X / 20 / \$ 21 \cdot$ doi: $10.1117 / 12.2555756$ 


\section{EXPERIMENTAL}

\subsection{Azopolymer thin films deposition and thickness measurement}

In our experiments we use the amorphous azopolymer poly[1-[4-(3-carboxy-4-hydroxyphenylazo) benzenesulfonamido]1,2-ethanediyl, sodium salt], or PAZO. The polymer is supplied by Sigma Aldrich and is used without further purification. Its chemical structure and spectrum of absorbance are shown in Fig. 1.

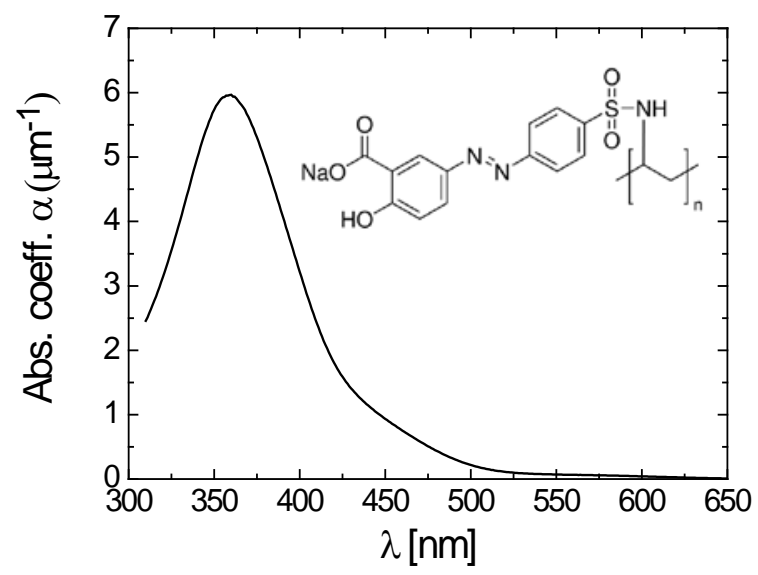

Figure 1. Spectrum of absorption and chemical structure of the azopolymer PAZO (adapted from Ref. 25).

Two series of thin film samples were prepared for the holographic experiments using the spin-coating technique. For the first series, PAZO was dissolved in pure distilled water and for the second - in methanol. In order to obtain films with different thickness for both series, we have varied the concentration of the solutions $C$, as well as the rotation speed $R$ during the spin-coating process. The specific values of $C$ and $R$ for each layer are given in Table 1 in the "Results and discussion" section. All samples are prepared using $200 \mu \mathrm{L}$ of the solution, the spin-coating time is $30 \mathrm{~s}$ and the substrates are preliminary cleaned BK7 glass slides. After drying overnight to ensure that the solvent is completely evaporated, the thickness of the samples is determined by an optical thin films analyzer (Filmetrics F20).

\subsection{Recording of the polarization holographic gratings}

The optical scheme used to inscribe polarization holographic gratings on the thin film samples is presented in Fig. 2. A He-Cd gas laser (Kimmon Koha) emmiting at $442 \mathrm{~nm}$ was used as recording laser.

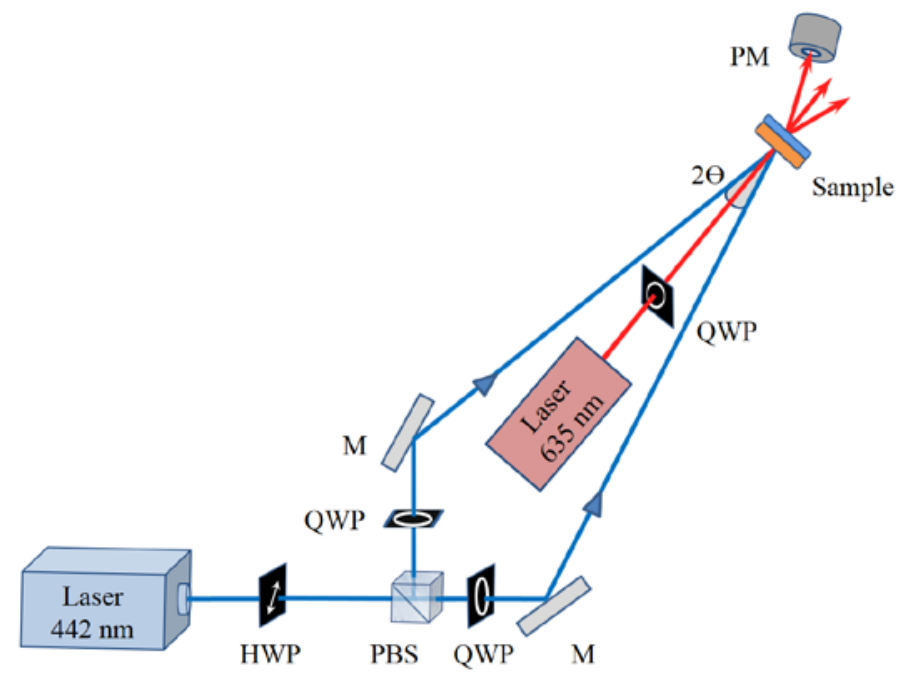

Figure 2. Setup for polarization holographic recording: HWP - half-wave plate, PBS - polarizing beam splitter, QWP quarter-wave plate, $\mathrm{M}$ - mirror, $\mathrm{PM}$ - power meter. 
This wavelength was selected, as according to our recent study ${ }^{26}$, highest birefringence $(\Delta n \approx 0.08)$ is obtained in PAZO at $442 \mathrm{~nm}$. Furthermore, this laser provides sufficient coherence length required for polarization holography. The beam is split in two beams with equal intensities by a half-wave plate and polarization beam splitter and their polarizations are set to left and right circular using quarter-wave plates. The two beams recombine on the sample at recording angle $2 \theta=20^{\circ}$, corresponding to grating period $\Lambda \approx 1.3 \mu \mathrm{m}$. Their total intensity is about $1000 \mathrm{~mW} / \mathrm{cm}^{2}$. A DPSS laser at 635 $\mathrm{nm}$ (B\&W Tek Inc.) is used as a probe laser, as its wavelength is outside the absorption band of the azopolymer PAZO, as seen in Fig. 1. Its polarization is set to right circular by an appropriate quarter-wave plate. In order to obtain the kinetics of the diffraction efficiency (DE), the diffracted power in the +1 order is measured in real time during the inscription of the grating by a computer operated power meter (PM100D, Thorlabs). The value of DE is given by the ratio of the diffracted power and the power of the probe laser beam incident on the grating.

\subsection{AFM analysis of the surface relief gratings}

To analyze the surface relief gratings formed during the polarization holographic recording, surface scans of the recorded areas were made using atomic force microscope (MFP 3D Scanning Probe Microscope, Asylum Research). The height $h$ of the surface relief is defined as the vertical distance between the highest and the lowest point of the topographic grating.

\section{RESULTS AND DISCUSSION}

Two series of samples were prepared according to the procedure described in Section 2.1. For the first series, solutions of PAZO in methanol were prepared with concentrations in the range $(25-100) \mathrm{mg} / \mathrm{mL}$. The rotation speed during spin coating was varied from 500 to $1500 \mathrm{rpm}$ and the resulting thicknesses of the samples are in the range (280-1550) $\mathrm{nm}$. The second series of azopolymer thin films was prepared from water solutions of PAZO with $C=(75-125) \mathrm{mg} / \mathrm{mL}$ and constant rotation speed $1500 \mathrm{rpm}$. The thicknesses in this series vary from $330 \mathrm{~nm}$ to $660 \mathrm{~nm}$.

Using the optical setup described in Section 2.2, we have recorded polarization holographic gratings in the samples from both series. The diffraction efficiency was monitored in real time in order to establish the moment when saturation is reached. The kinetic curves of the DE are shown in Fig. 3 for the samples with highest diffraction efficiency from both series - PAZO/methanol and PAZO/water.
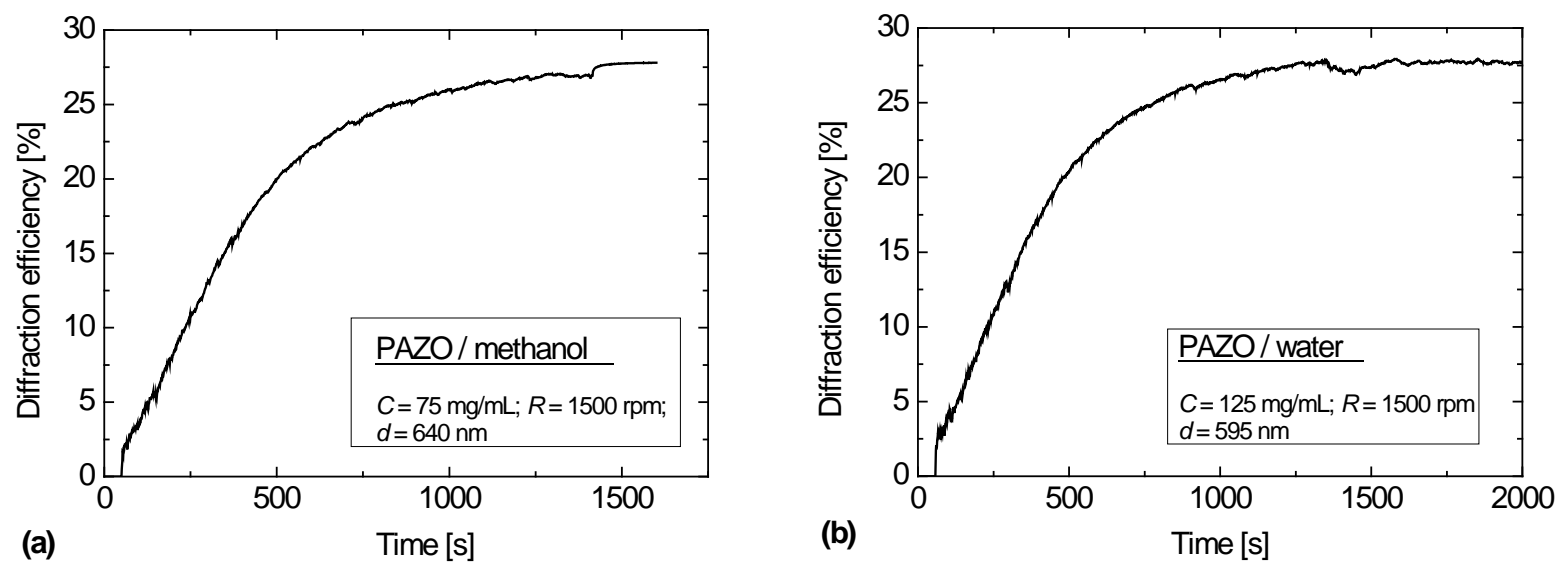

Figure 3. Diffraction efficiency kinetics in the +1 diffraction order during the holographic recording for the thin film samples: (a) PAZO/methanol sample, $d=640 \mathrm{~nm}$; (b) PAZO/water sample, $d=660 \mathrm{~nm}$.

As seen in Fig. 3, the kinetic behavior of the DE in the two types of samples is similar. We can define a response time as the recording time needed to reach $80 \%$ of the saturated $\mathrm{DE}$. In this case, the response time for the $\mathrm{PAZO} / \mathrm{methanol}$ sample in Fig. 3(a) is $560 \mathrm{~s}$, and for the PAZO/water sample in Fig. 3(b) it is $520 \mathrm{~s}$. This response time is much higher than the birefringence response time at this wavelength and laser intensity ${ }^{26}$, which is $15 \mathrm{~s}$. This implies that the volume polarization grating is formed in the initial stage of recording and the rest of the recording time is required for the formation of the surface relief grating. 

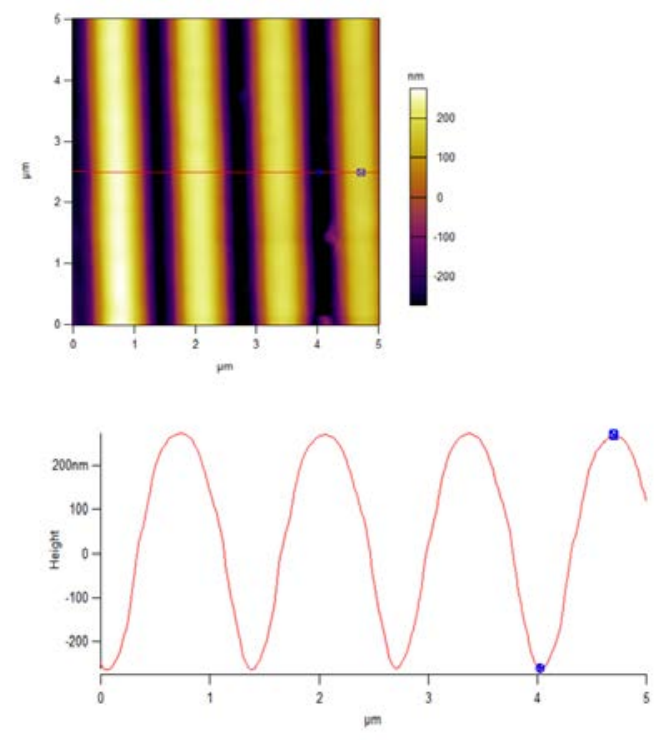

(a)

$$
\begin{aligned}
& 0 x=133 \mu \mathrm{mm} \\
& 0 y=28 \mathrm{~nm}
\end{aligned}
$$
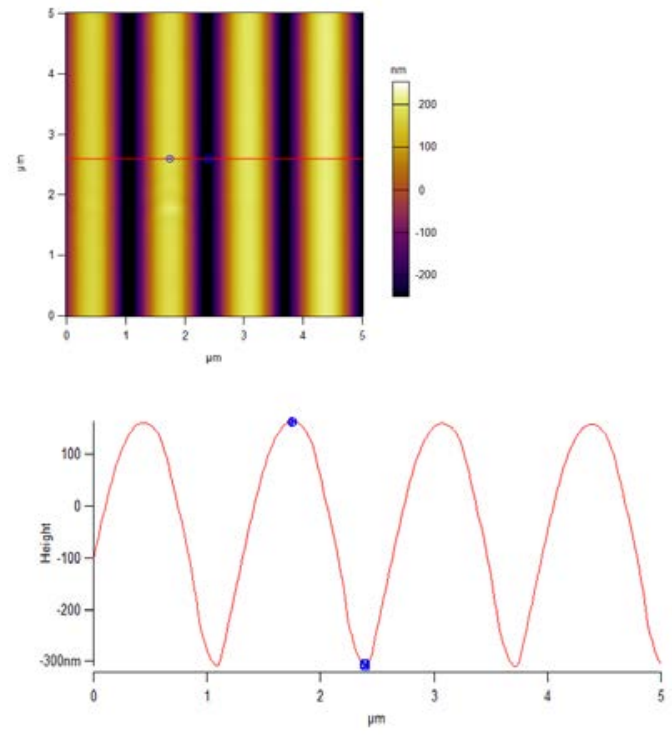

(b)

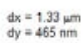

Figure 4. Atomic force microscope (AFM) images of the surface relief gratings formed in the thin film samples: (a) PAZO/methanol sample ( $d=625 \mathrm{~nm}), h=528 \mathrm{~nm}$; (b) PAZO/water sample $(d=595 \mathrm{~nm}), h=465 \mathrm{~nm}$.

The experimental data for all samples prepared and recorded within this experiment are summarized in Table 1 below. The dependence of the maximal (saturated) diffraction efficiency on the thickness of the sample is graphically presented in Fig. 5 for both PAZO/methnol and PAZO/water thin film series. A spline curve is plotted through the experimental data points to better illustrate the trends.

Table 1. Summary of the prepared azopolymer thin films and parameters of the gratings, inscribed in them.

\begin{tabular}{|c|c|c|c|c|c|}
\hline № & $\begin{array}{c}\text { Concentration } \\
\boldsymbol{C}[\mathbf{m g} / \mathbf{m L}]\end{array}$ & $\begin{array}{c}\text { Rotation speed } \\
\boldsymbol{R}[\mathbf{r p m}]\end{array}$ & $\begin{array}{c}\text { Film thickness } \\
\boldsymbol{d} \text { [nm] }\end{array}$ & $\begin{array}{c}\text { Diffraction efficiency } \\
\text { DE [\%] }\end{array}$ & $\begin{array}{c}\text { SRG height } \\
\boldsymbol{h} \text { [nm] }\end{array}$ \\
\hline \multicolumn{5}{|c|}{ PAZO / methanol } \\
\hline 1 & 25 & 500 & 280 & 7.0 & 175 \\
\hline 2 & 50 & 700 & 350 & 13.1 & - \\
\hline 3 & 50 & 600 & 450 & 25.7 & 169 \\
\hline 4 & 50 & 500 & 625 & 25.5 & 528 \\
\hline 5 & 75 & 1500 & 640 & 27.8 & 321 \\
\hline 6 & 100 & 1400 & 1550 & 27.8 & - \\
\hline \multicolumn{5}{|c|}{ PAZO / water } \\
\hline 7 & 75 & 1500 & 330 & 9.0 & - \\
\hline 8 & 100 & 1500 & 450 & 18.3 & - \\
\hline 9 & 125 & 1500 & 595 & 27.7 & 465 \\
\hline 10 & 125 & 1500 & 660 & 25.9 & 139 \\
\hline
\end{tabular}

As seen, for lower film thicknesses the $\mathrm{DE}$ value is higher for the PAZO/methanol series, however a convergence is observed for thickness above $600 \mathrm{~nm}$. It should also be noted, that the DE of the sample with highest thickness from the $\mathrm{PAZO} /$ methanol series $(d=1550 \mathrm{~nm})$ is the same as the DE of the thinner layer $(d=640 \mathrm{~nm})$. This indicates that for the given setup this is the highest achievable diffraction efficiency. 


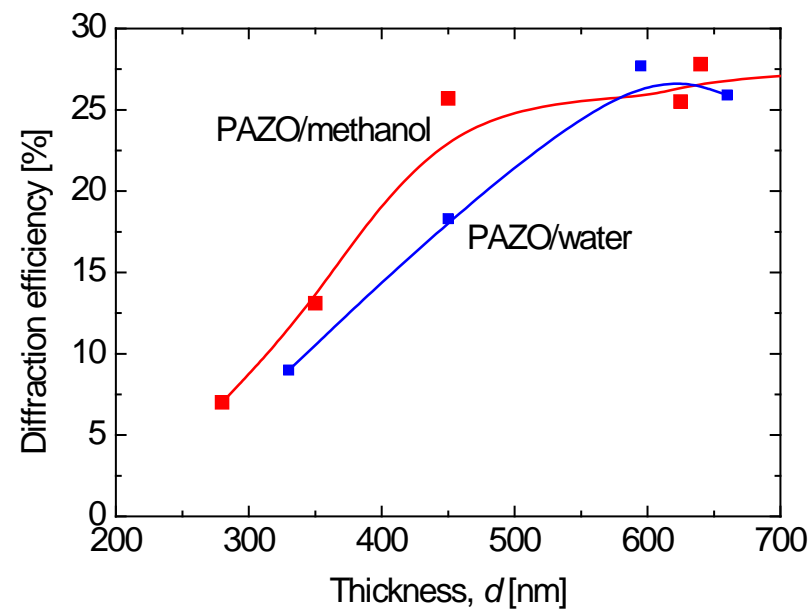

Figure 5. Dependence of the diffraction efficiency on the thickness of the azopolymer thin films for the series $\mathrm{PAZO} / \mathrm{methanol}$ and PAZO/water.

\section{CONCLUSIONS}

Two series of thin film samples were prepared from the azopolymer PAZO - one using methanol as a solvent, and second using pure water as solvent. Polarization holographic gratings were recorded in these samples with beams with orthogonal circular polarizations (RCP and LCP) at angle $2 \theta=20^{\circ}$ and $442 \mathrm{~nm}$ wavelength of the recording laser. Diffraction efficiency of $27.8 \%$ was obtained. Our experiments indicate that for thickness of the sample below $600 \mathrm{~nm}$, the diffraction efficiencies of the PAZO/methanol series is higher compared to the samples from the PAZO/water series. Surface relief gratings with significant height have been obtained in both series. More specifically, $h=528 \mathrm{~nm}$ has been reached, comparable with the sample thickness $(d=625 \mathrm{~nm})$. In addition, higher DE values have been obtained compared to our previous work ${ }^{27}$, where under similar conditions $\left(2 \theta=20^{\circ}, \lambda_{\text {rec }}=442 \mathrm{~nm}, d=850 \mathrm{~nm}\right)$, the maximal value of DE was $9.8 \%$.

\section{ACKNOWLEDGEMENT}

Financial support provided by National Science Fund of Bulgaria under the grant ДН 08/10 is gratefully appreciated. Research equipment of distributed research infrastructure INFRAMAT (part of Bulgarian National roadmap for research infrastructures) supported by Bulgarian Ministry of Education and Science under contract D01-284/17.12.2019 was used in this investigation.

\section{REFERENCES}

[1] Todorov, T., Nikolova, L. and Tomova, N., "Polarization Holography 1. A new high-efficiency organic material with reversible photoinduced birefringence,” Appl. Opt. 23, 4309-4312 (1984).

[2] Hvilsted, S., Andruzzi, F., Kulinna, C., Siesler, H. W. and Ramanujam, P. S., "Novel side-chain liquid crystalline polyester architecture for reversible optical storage," Macromolecules 28, 2172-2183 (1995).

[3] Nedelchev, L., Matharu, A. S., Hvilsted, S. and Ramanujam, P. S., "Photoinduced anisotropy in a family of amorphous azobenzene polyesters for optical storage,” Appl. Opt. 42, 5918-5927 (2003).

[4] Castro-Pérez, C. V., Trejo-Carbajal, N., Rodríguez-González, R. J., Larios-López, L., Felix-Serrano, I. and Navarro-Rodríguez, D., "Liquid crystal and photo-induced properties of polymers carrying pyridylazobenzene groups and iodopentafluorobenzene rings self-assembled through halogen bond,” J. Fluor. Chem. 222-223, 9099 (2019). 
[5] Kim, D. Y., Li, L., Jiang, X.L., Shivshankar, V., Kumar, J. and Tripathy, S. K., "Polarized laser induced holographic surface relief gratings on polymer films," Macromolecules 28, 8835-8839 (1995).

[6] Berendt, J., Teixeira, J. M., García-García, A., Raposo, M., Ribeiro, P.A., Dubowik, J., Kakazei, G. N. and Schmool, D. S., "Tunable magnetic anisotropy in permalloy thin films grown on holographic relief gratings," Appl. Phys. Lett. 104, 082408 (2014).

[7] Pagliusi, P., Audia, B., Provenzano, C., Piñol, M., Oriol, L. and Cipparrone, G., "Tunable Surface Patterning of Azopolymer by Vectorial Holography: The Role of Photoanisotropies in the Driving Force,” ACS Appl. Mater. Interf. 11 (37), 34471-34477 (2019).

[8] Nedelchev, L., Todorov, T., Nikolova, L., Petrova, Tz., Tomova, N. and Dragostinova, V., "Characteristics of high-efficient polarization holographic gratings," Proc. SPIE 4397, 338-342 (2001).

[9] Martinez-Ponce, G., Petrova, Ts., Tomova, N., Dragostinova, V., Todorov, T. and Nikolova, L., "Bifocalpolarization holographic lens,” Opt. Lett. 29, 1001-1003 (2004).

[10] Ramanujam, P. S., Dam-Hansen, C., Berg, R. H., Hvilsted, S. and Nikolova, L., "Polarisation sensitive optical elements in azobenzene polyesters and peptides,” Opt. Lasers Eng. 44, 912-925 (2006).

[11] Natansohn, A. and Rochon, P., "Photoinduced motions in azo containing polymers," Chem. Rev. 102, 41394175 (2002).

[12] Nikolova, L. and Ramanujam, P.S., [Polarization Holography], Cambridge University Press, Cambridge (2009).

[13]Wang, X., [Azo Polymers: Synthesis, Functions and Applications], Springer-Verlag, Berlin (2017).

[14]Cipparrone, G., Pagliusi, P., Provenzano, C. and Shibaev, V. P., "Polarization holographic recording in amorphous polymer with photoinduced linear and circular birefringence,” J. Phys. Chem. B 114, 8900-8904 (2010).

[15]Zhao, F., Wang, Ch., Qin, M., Zeng, P. and Cai, P., "Polarization holographic gratings in an azobenzene copolymer with linear and circular photoinduced birefringence,” Opt. Commun. 338, 461-466 (2015).

[16] Viswanathan, N., Balasubramanian, S., Li, L., Tripathy, S. and Kumar, J., "A detailed investigation of the polarization-dependent surface-relief-grating formation process on azo polymer films,” Jpn. J. Appl. Phys. 38, 5928-5937 (1999).

[17] Rochon, P., Batalla, E. and Natansohn, A., "Optically induces surface gratings on azoaromatic polymer films," Appl. Phys. Lett. 66, 136-138 (1995).

[18]Kim, D. Y., Lee, T. S., Wang, X., Jiang, X. L., Li, L., Kumar, J. and Tripathy, S. K., "Photofabrication of surface relief gratings on azobenzene polymer films,” Proc. SPIE 2998, 195-204 (1997).

[19] Ferreira, Q., Gomes, P., Raposo, M., Giacometti, J. A., Oliveira, O. and Ribeiro, P., "Influence of Ionic Interactions on the Photoinduced Birefringence of Poly[1-[4-(3-Carboxy-4-Hydroxyphenylazo) BenzeneSulfonamido]-1,2-Ethanediyl, Sodium Salt] Films,” J. Nanosci. Nanotechnol. 7, 2659-2666 (2007).

[20] Raposo, M., Ferreira, Q., Monteiro Timóteo, A. R., Ribeiro, P. and Botelho do Rego, A. M., "Contribution of counterions and degree of ionization for birefringence creation and relaxation kinetics parameters of PAH/PAZO films,” J. Appl. Phys. 118, 114504 (2015).

[21] Frascella, F., Angelini, A., Ricciardi, S., Pirri, F. and Descrovi, E., "Surface-relief formation in azopolyelectrolyte layers with a protective polymer coating," Opt. Mater. Exp. 6(2), 444-450 (2016).

[22] Zagalo P., Magalhães-Mota G., Sério S., Ribeiro P. and Raposo M., "Electric-field Induced Birefringence in Azobenzene Thin Films," Proceedings of the 5th International Conference on Photonics, Optics and Laser Technology - Volume 1: AOMat, 381-385 (2017).

[23] Nedelchev, L., Mateev, G., Otsetova, A., Nazarova, D. and Stoykova, E., "Optimization of deposition of thin photoanisotropic films for holographic data storage,” Int. J. Inf. Theor. Appl. 25, 245-254 (2018).

[24] Stoykova, E., Blagoeva, B., Nedelchev, L. and Nazarova, D., "Temperature dependence of the drying process in polymer solutions observed by dynamic speckle detection,” Proc. SPIE 11207, 112071S (2019).

[25] Berberova, N., Nazarova, D., Nedelchev, L., Blagoeva, B., Kostadinova, D., Marinova, V. and Stoykova, E., "Photoinduced variation of the Stokes parameters of light passing through thin films of azopolymer-based hybrid organic/inorganic materials,” J. Phys.: Conf. Ser. 700, 012032 (2016).

[26] Nedelchev, L., Ivanov, D., Blagoeva, B. and Nazarova, D., "Optical anisotropy induced at five different wavelengths in azopolymer thin films: Kinetics and spectral dependence,” J. Photochem. Photobiol. A, 376, 1-6 (2019).

[27] Nedelchev, L., Ivanov, D., Berberova, N., Strijkova, V. and Nazarova, D., "Polarization holographic gratings with high diffraction efficiency recorded in azopolymer PAZO,” Opt. Quant. Electron. 50:212 (2018). 